\title{
Influencia de la retórica musical de Los Beatles en los "jingles" y en la publicidad
}

\section{Influence of The Beatles' musical rhetoric in jingles and advertising}

\author{
Antón Álvarez Ruiz
}

Profesor Titular Interino. Facultad de Ciencias de la Información, UCM

Fecha de recepción: 15 de noviembre de 2012

Fecha de revisión: 21 de enero de 2013

Para citar este artículo: Álvarez Ruiz, A. (2013): Influencia de la retórica musical de Los Beatles en los "jingles" y en la publicidad, Icono 14, volumen 11 (1), pp. 139-161. doi: 10.7195/ri14.v11i1.558 


\section{Resumen}

Este artículo explora de qué manera los jingles publicitarios se han visto influidos por la música de The Beatles. Para ello, utiliza una metodología descriptiva, comparativa y diacrónica. Tras estudiar brevemente la historia e importancia de los jingles, analizando algunas características de las canciones de The Beatles y su semejanza con los jingles publicitarios. En las canciones mencionadas se detallan las principales figuras retóricas encontradas, tanto en los textos como en las frases musicales y su influencia en la música publicitaria. También se estudia la única pieza publicitaria conocida realizada por The Beatles en su carrera. Por último, se analiza la posterior utilización de sus canciones y su imagen en varias campañas muy significadas, realizadas por la publicidad comercial en las últimas décadas.

\section{Palabras clave}

Beatles y publicidad - jingles y música pop - música y publicidad

\section{Abstract}

This article explores the way in which advertising jingles have been influenced by the music of The Beatles. For this purpose, descriptive, comparative and diachronic methodology was used. After briefly studying the history and importance of advertising jingles, we have analyzed some of the characteristics of The Beatles songs and their similarities to advertising jingles. In the songs mentioned we highlight the main rhetorical figures found both in the lyrics and musical phrases and their influence on advertising jingles. The article also studies the solely musical theme recorded by the Beatles in their professional career for advertising purposes. Lastly, the subsequent use of their songs and images in several significant campaigns found in commercial advertisement in the last decades are analyzed.

\section{Key Words}

Beatles and advertising - jingles and pop music - music and advertising 


\section{Introducción y objetivos}

En este artículo deseamos estudiar de qué manera las figuras y los recursos retóricos presentes en las canciones de The Beatles han influenciado los jingles publicitarios. Sabemos que las figuras retóricas están presentes tanto en la música popular como en la música culta desde su origen. Pero el hecho de que The Beatles hicieran un uso extensivo y concentrado de estas figuras, sumado a que las canciones publicitarias han pretendido un efecto similar para facilitar el recuerdo de las marcas y vincularlas a los consumidores, ha permitido espacios de convergencia entre ambas actividades, propiciando esta influencia. Igualmente, queríamos analizar ciertas relaciones observadas entre la música pop y la publicidad $\mathrm{y}$, por extensión, la significación retórica que tiene la propia presencia de celebrities del pop y del rock en la comunicación persuasiva.

\section{Metodología}

Se ha utilizado un enfoque marcadamente cualitativo, siguiendo un procedimiento analítico, descriptivo y evolutivo que, según creemos, es el que exigía el tema abordado. Para ello, se ha estudiado en primer lugar la historia y evolución de los jingles publicitarios; también se ha analizado la práctica totalidad de las canciones de The Beatles y se han seleccionado para su comentario los temas que presentan figuras retóricas muy habituales en los jingles, categorizándolas en base a sus expresiones retóricas. En un nivel subsiguiente, se ha observado cómo se produjo la incorporación de las canciones de The Beatles y de otros grandes artistas a campañas publicitarias de gran repercusión pública -hecho ocurrido precisamente cuando los jingles comenzaban a ser sustituidos por temas originales- analizando las interrelaciones mutuas creadas. Como colofón, también observamos los efectos retóricos y comunicacionales conseguidos cuando se produce la incorporación de los propios grupos y cantantes en las campañas de publicidad. 


\section{Un soporte eficaz para los mensajes}

\section{publicitarios}

Uno de los muchos elementos propios y originales que ha creado la industria publicitaria son los jingles, esas canciones sencillas, sonoras y muy pegadizas, que consiguen que el nombre, el eslogan y las ventajas de los productos se graben en nuestro recuerdo de forma persistente, a veces incluso contra nuestra voluntad, y orienten en una determinada dirección nuestras pautas de consumo. Técnicamente, podemos definir los jingles como "una pieza de música compuesta especialmente para acompañar a los anuncios de publicidad y que se caracteriza por ser de corta duración y fácil de recordar" (Hoyuela y Lázaro, p. 142). Y resulta llamativo que, a pesar de su amplio uso y de su popularidad, apenas han suscitado estudios fundados y reflexiones críticas (Fernández Gómez 2002, p. 401; Sala, p. 12). Y, aunque últimamente hayan aparecido algunos escritos científicos sobre el tema, estos se dedican mayormente a clasificar los jingles en función de sus diferentes tipologías y usos en lugar de analizar lo que creemos resultaría más interesente, como son su función, su contenido o sus propuestas retóricas.

Quizás los jingles no han llamado especialmente la atención de los investigadores porque en la comunicación persuasiva la música suele jugar un papel trascendente pero poco visible (Sánchez Guzmán, p. 173). Por eso, cuando presenciamos una pieza audiovisual, percibimos la imagen con mayor intensidad que la banda sonora. Y cuando escuchamos una pieza de audio solemos proyectar nuestra atención hacia el texto antes que hacia la música.

Aunque en las últimas décadas el uso de jingles propiamente dichos está perdiendo protagonismo ante el uso de temas originales y de versiones (Espadalé, p. 289), aquellos han jugado durante casi un siglo un papel muy importante en la actividad publicitaria por su capacidad para ejercitar la recordación de la marca, sus propiedades y valores (Ferrer, p. 160; Sala, p. 12 y 43) porque, al combinar las "tres erres" -ritmo, rima y repetición- se consiguen niveles de memorización superiores a los que logra una banda sonora sin música (Palencia-Lefler, p. 93-4), motivo por el que nueve de cada diez anuncios audiovisuales llevan música (Espadalé, p. 289).

Los jingles publicitarios aparecieron prácticamente con la radio comercial a primeros de los años 20 (Wikipedia, 2010) demostrando una vez más la facilidad que

ICONO14 | Año 2013 Volumen 11 Nº 1 | ISSN: 1697-8293 | DOI: ri14.v11i1.558 
ostenta la publicidad para filtrarse en los nuevos medios y canales de comunicación. Y en esos primeros años de historia de la radio los publicitarios descubrieron rápidamente que eran mucho más eficaces los mensajes que iban ubicados en una canción que los que simplemente eran leídos por los locutores.

\section{Cuando la música representa el alma de la}

\section{marca}

Con el tiempo, los jingles y otras melodías de anuncios han evolucionado notablemente, produciéndose en ellos un marcado proceso de estilización creativa. Y se han comprometido a tal nivel con la imagen de algunas marcas que han llegado a formar parte sustancial de los valores de estas promueven (Aizcorbe, p. 10). La campaña considerada de mayor éxito en la historia de la publicidad y que ostenta el récord de duración, los cowboys de Marlboro creados por la agencia Leo Burnett en 1954 (Souter y Newman, p. 234), se apoya desde sus inicios en la excelente banda sonora de "Los siete magníficos" de Elmer Bernstein (Sturges). Su evocadora música contribuye a visualizar el mundo de libertad y rudeza de los cowboys, erigiéndose en un pilar central en la construcción del imaginario de la marca (Marlboro 1963), y ha sido el objeto específico de algunas campañas de publicidad realizadas por la propia marca (Marlboro 1967).

En España, encontramos un caso comparable en términos de protagonismo y durabilidad: el jingle de la 0nce (Organización Nacional de Ciegos de España) titulado “la ilusión de todos los días". Emitido por primer vez en 1983 (Álvarez. p. 91; 0nce) forma parte sustancial del carácter popular y positivo de la marca, especialmente en el medio radio, donde está íntimamente asociado a la lectura del cupón premiado. El tema musical de la Once -que, igual que el de Marlboro, constituye lo que se denomina "logo musical" o "sintonía corporativa", pues al espectador le basta oír algunas notas para recordar inmediatamente la marca anunciada- ha sido utilizado durante décadas hasta hoy en día, actualizado periódicamente con diferentes arreglos, y ha logrado una pervivencia superior al jingle más recordado en nuestro país, según consideración de los expertos: el famoso tema "Yo soy aquel negrito" de Cola-Cao, iniciado a finales de los años 50 y que alcanzó una notoriedad muy ele- 
vada en la década siguiente (Summers, p. 33-34; Palencia-Lefler, p. 90; Cola-Cao).

Dentro de su actuación, tan modesta como eficaz, los jingles se apoyan en la simplicidad de la música y de la letra para lograr la fácil recordación que ya hemos mencionado (Lorente, p. 109) y, partiendo de un esquema muy básico de composición, hablan un lenguaje directo y cercano que conecta rápidamente con el público porque utiliza los mismos códigos de las personas a las que va dirigido (Fernández Gómez; Muela, p. 195). Seguramente es en esta inmediatez, en la aparente sencillez con la que consiguen facilitar la recordación del nombre y las propiedades de la marca asociadas a un fraseo musical contagioso, donde radica su principal cualidad.

\section{Los jingles en la era de la televisión}

A finales de los años 50 y primeros de los 60, cuando la televisión vivió una rápida expansión, los jingles se popularizaron en este nuevo medio, alcanzando notable cotas de perfección e incrementando su eficacia. Ahora que la música publicitaria tenía la posibilidad de trabajar conjuntamente con la imagen visual, comenzó una nueva etapa en la persuasión comercial, que ofrecería piezas más elaboradas y atractivas, explotando intensivamente los recursos combinados del audio y del vídeo. Fue también en esta época cuando comenzó a hacerse muy famoso un grupo inglés llamado The Beatles que componía un tipo de rock muy personal, sencillo y directo, apoyado en melodías brillantes y fáciles de recordar.

Además de muchas otras influencias musicales, resulta evidente que la forma de componer de The Beatles se vio influenciada inicialmente por los jingles publicitarios. Y de hecho, algunos miembros del grupo han admitido repetidas veces que obtenían la inspiración de cuanto les rodeaba, utilizando técnicas como ojear los periódicos y la televisión, escuchar la radio o ver anuncios (The Beatles, 2000, p. 97), igual que ocurría con otros músicos de su época que intentaban conseguir de esta manera una aproximación más informal a la composición musical (Miles, p. 71). Otra grandes figuras de la canción popular, contemporáneas de The Beatles como Paul Simon, o posteriores como U2, han incorporado eslóganes de conocidas campañas de publicidad en algunos de sus temas para criticar el consumismo ("The Big Bright Green Pleasure Machine" de Simon, 1966; "Zooropa” de U2, 1993). Por

ICONO14 | Año 2013 Volumen 11 NN $^{\circ}$ | ISSN: 1697-8293 | DOI: ri14.v11i1.558 
todo ello, parece justificado suponer que los jingles de publicidad influenciaron a The Beatles en su época rock y pudieron contribuir a preparar su evolución hacia la música pop, que sería su gran aportación a la historia de la música popular. Pero lo que nos parece totalmente incuestionable -aunque nunca lo hayamos visto mencionado- es la influencia que los propios Beatles ejercieron, a su vez, sobre los jingles publicitarios. Y creemos que es por este motivo por lo que resulta fácil encontrar una larga variedad de lugares comunes entre los jingles y las canciones de The Beatles, especialmente si nos basamos en las figuras retóricas utilizadas en ambos discursos musicales, como detallamos a continuación.

\section{Los jingles y The Beatles: influencias y puntos}

\section{de encuentro}

\subsection{Tono optimista}

Igual que un buen jingle, las canciones de The Beatles muestran un tono alegre y dinámico. Quizás esta característica sea una de las más significativas de la música de The Beatles pues este grupo sabía transmitir a sus canciones una elevada dosis de confianza en sí mismas, como hacen los productos y las marcas bien asentadas. Uno de sus primeros éxitos, "Please, please me" nos ofrece un buen ejemplo; también temas como "I feel fine" o "Drive my car" transpiran este optimismo contagioso que les permite conectar con el público para hacerse familiares rápidamente, efecto que busca cualquier marca.

\subsection{Melodías condensadas}

Las primeras canciones de The Beatles son sencillas, cortas y contundentes; musicalmente están bastante condensadas y concentran en pocas notas su melodía. Entran directamente en el tema musical y pasan enseguida al estribillo sin dar más rodeos. Este procedimiento de buscar atajos y de plantear directamente la melodía esencial del tema, le permitió a The Beatles explorar en alguna canción soluciones técnicas bastante originales para su época como empezar directamente por el es-

DOI: ri14.v11i1.558 | ISSN: 1697-8293 | Año 2013 Volumen 11 No 1 | ICONO14 
tribillo, lo que ocurre en "She loves you", "Strawbewrry fields forever" o "We can work it out", constituyendo esta última un buen ejemplo de expolición. Ambas características de condensar la melodía y de acceder directamente a los más pegadizo del tema son propiedades que suelen encontrarse en todo buen jingle publicitario.

\subsection{Arranques llamativos, finales redondos}

Mención aparte merecen los inicios y finales de las canciones, dos partes que suelen estar cuidadosamente elaboradas en todo jingle. Siguiendo el mismo criterio, una vez compuesta la melodía, The Beatles trabajaban a fondo los arreglo musicales del principio y del final de cada tema, para hacerlos estilísticamente más limpios y pulcros.

Muchas de sus canciones podrían servirnos como ejemplo de esta característica, pero quizás las más lograda en este aspecto sea "Help!" Si revisamos su comienzo (ver The Beatles, 1965, desde 0:0 a 0:08; consultar la letra en el cuadro ${ }^{0}{ }^{1}$ ) observaremos un arranque contundente y llamativo, elaborado para atrapar la atención del espectador más indolente.

\section{Cuadro 1. Canción "Help"}

\begin{tabular}{|l|}
\hline Comienzo de la canción \\
\hline Help!, I need somebody, \\
Help!, not just anybody, \\
Help!, you know I need someone... Heeelp! \\
\hline Final de la canción \\
\hline Won't you please, please help me? Help me, help me-ee, uh-uh. \\
\hline
\end{tabular}

Fuente: The Beatles, 1965

Y luego podemos prestar atención al final (The Beatles 1965, ver desde 2:01 a 2:10; letra en el cuadro $n^{0} 1$ ). Si el comienzo del tema ya es notable, la terminación es realmente brillante: durante la canción el ritmo es bastante rápido, pero a unos segundos del final se ralentiza y termina dejando una especie de vibración en el aire que prolonga subjetivamente la duración real del tema musical. Este recurso es un efecto muy buscado también por los jingles para dilatar su eficacia y estirar subjetivamente su duración. Finales redondos de este tipo pueden encon- 
trarse en otras canciones de The Beatles como "Eight days a week", "Penny Lane" o "Lady Madonna". Suponían una importante innovación musical y una muestra de la inquietud creativa de The Beatles, en una época en la que resultaba habitual terminar las canciones repitiendo varias veces el estribillo mientras el volumen descendía en un previsible fade out, procedimiento que, por otra parte, consumía más tiempo.

\subsection{Repeticiones y otros juegos retóricos}

Tanto los jingles como las canciones de The Beatles han desplegado a lo largo de su producción una panoplia muy amplia de recursos retóricos, aplicando reiteradamente un buen número de las figuras de la oratoria ortodoxa desarrolladas y clasificadas en la Grecia clásica.

Las repeticiones son el recurso retórico más utilizado, no sólo en el texto sino -y muy especialmente- en las frases musicales. No cabe duda de que la repetición reiterada -el denominado ostinato en lenguaje musical culto- constituye la base del rock y del pop. Y esa característica es, en gran medida, la que consigue que las canciones de estos géneros sean tan fáciles de escuchar y de retener, propiedad que en lenguaje popular conocemos con la expresión "es un tema pegadizo". Pero en The Beatles este recurso estilístico alcanzó un particular virtuosismo en un amplio número de piezas, como podemos comprobar repasando los excelentes riffs de canciones como "I feel fine", “Day tripper", "And your bird can sing”, "Paperback writter", "Ticket to ride" o "Drive my car". Además de sus funciones puramente musicales, el uso de la repetición consigue que el público memorice al menos una parte significativa de las canciones prácticamente durante la primera audición.

Así como los jingles repiten varias veces el nombre de la marca anunciada, su eslogan o su principal beneficio, The Beatles solían repetir el título de la canción varias veces, en ocasiones al principio del tema -realizando una reiteración o anáfora, como en el caso que hemos visto de "Help"- o más frecuentemente al final del tema para facilitar su recuerdo. En otras ocasiones el recurso repetitivo utilizado para cerrar la canción era volver a recordar la frase musical del comienzo, cerrando el círculo, como en "Eight days a week" y "Eleanor Rigby", constituyendo ambos temas claros ejemplos de epanadoplosis musical. Esta última canción también nos ofrece sendos ejemplos de etopeya y de hipotiposis, aplicados a la propia Eleanor

DOI: ri14.v11i1.558 | ISSN: 1697-8293 | Año 2013 Volumen 11 No 1 | ICONO14 
Rugby y al padre McKenzie, a cuyas vidas se refiere la letra.

Otras veces la repetición encierra una intención y un efecto más originales, como en "I wanna hold your hand": la frase "I can't hide, I can't hide..." se escucha varias veces seguidas, ofreciendo una sutil paradoja que bromea con el espectador y estimula su interés, al dar la impresión de que el disco se ha rayado y ha entrado en un bucle; algo muy frecuente en la época de los microsurcos de vinilo.

Los juegos de palabras y las expresiones de doble lectura, especialmente las antítesis y las homonimias, también fueron muy utilizados por The Beatles (Dister, p. 17 y 24; Miles, p. 75). Podemos comenzar ocupándonos del nombre del grupo que ofrece un ingenioso ejemplo de paronomasia porque, al margen del significado principal ("The Beatles = "Los Rítmicos") contiene otra acepción más burlona sugerida por su sonido ("The Beetles" = "Los Escarabajos") (Dister, p. 26). También podríamos destacar que "The Beatles" además de un nombre muy acertado para un grupo musical constituye una excelente denominación de producto, que cumple con la mayoría de los requisitos que se le exigen a una marca en las pruebas de naming: resulta eufónico, corto, memorable, fácil de pronunciar, explicativo, descriptivo, fácilmente convertible en logotipo (ver gráfico $n^{0} 1$ ), etc.

Además del nombre y del logo, bastantes otras acciones del grupo podrían clasificarse como brillantes ejemplos de comunicación publicitaria y del aprovechamiento consciente de los medios con un fin persuasivo: su estudiada imagen con los flequillos, las portadas de sus discos, las vestimentas utilizadas, las ruedas de prensa -excelentes ejercicios de relaciones públicas-, la iniciativa de rodar películas de cine y videoclips, la transmisión internacional en directo de la grabación de un tema que se hizo famoso al instante ("All you need is love"), etc. Pero profundizar en estos campos excedería con mucho la propuesta de este escrito.

Siguiendo con los juegos de palabras, algunas canciones contienen notables

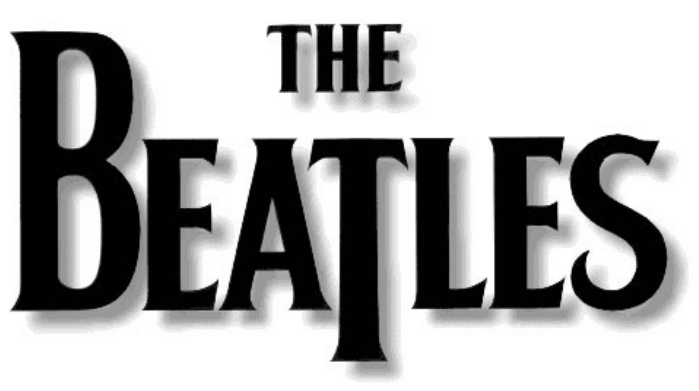

\section{Gráfico 1}

El famoso logo con la " $T$ " caída, utilizado en la primera época del grupo y realizado en 1963 por Ivon Arbiter y Eddie Stokes, subraya y refuerza la paronomaxia existente en el nombre

(Fuente: The Beatles Bible).

ICONO14 | Año 2013 Volumen 11 N 1 | ISSN: 1697-8293 | DOI: ri14.v11i1.558 
muestras de figuras retóricas en los títulos y en el texto, generalmente difíciles de traducir al castellano, aunque en el idioma inglés saben mantener el nivel de simplicidad del resto de las letras para permitir que el público en general, con independencia de su nivel cultural, pueda comprenderlas. Así ocurre con los oxímoron de "Please please me" ("Por favor, compláceme") y de "A hard day's night" ("La noche de un día agotador"), en el primer caso combinado con una aliteración y en segundo con una antítesis.

Asimismo, encontramos una hipotiposis sostenida en la alegre y vivaz descripción de las personas que pueblan el callejón del penique en "Penny Lane" -cuya letra está llena de divertidas licencias- , una llamativa paradoja en "Eight days a week" ("Ocho días a la semana", ver The Beatles, 2000, p. 159) o una hábil paronomasia en el estribillo de "She's living home" cuando el texto entrelaza de manera figurada la despedida de la hija que se escapa de casa y los reproches de sus padres, creando una especie de conversación entre sordos, en el pasaje musicalmente denominado "coros griegos" (ver McCartney, desde 1:06 a 1:20; letra en el cuadro $\left.n^{0} 2\right)$.

\section{Cuadro 2. Coros griegos de la canción "She's living home" (fragmento)}

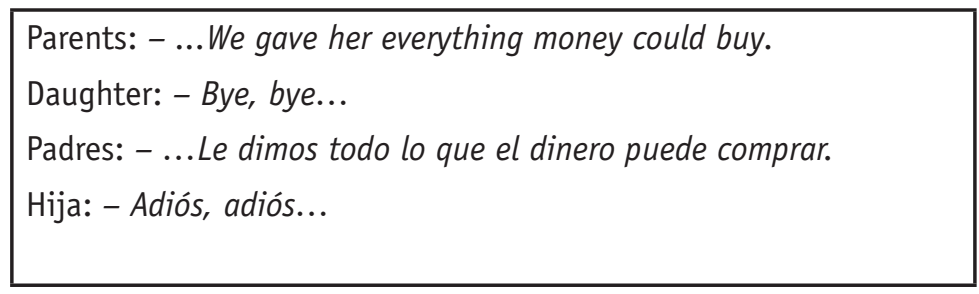

Fuente: McCartney

Estas frases juegan con la fonética de las palabras "buy" y "bye", que en inglés suenan exactamente igual. Es muy probable que esta canción inspirase una campaña publicitaria realizada en algún aeropuerto de Londres a finales de los años 80; se trataba de unos carteles que utilizando letras de gran tamaño decían simplemente "Buy, buy" (literalmente "compre, compre", aunque suena como "buen viaje") y que iban oportunamente firmados por las Duty Free Shops del propio ae- 
ropuerto. Estos coros griegos también ofrecen un ejemplo continuado de antítesis.

En la canción "Girl" encontramos sendas bromas de doble intención perfectamente dispuestas para el oyente atento. En primer lugar, si nos fijamos en el acompañamiento vocal de la variación musical, cantada pulcramente a tres voces, distinguiremos que dice "tit, tit, tit..." ("teta, teta, teta...") en lugar del habitual "dit, dit, dit" (The Beatles, 2000, pag. 196, Fontenot, 2011).

En segundo lugar, podemos escuchar el sonido de aspiración que aparece en el estribillo tras la palabra que da título a la canción y que muchos oyentes interpretaron en su día como un suspiro de deseo. Pero este recurso estilístico, que fue convenientemente enfatizado en las mezclas de la canción, pudo ser preparado por The Beatles como un mensaje encubierto para los fumadores de porros -y de hecho, suena como una fuerte aspiración dada a un cigarrillo- en una época en que esta actividad comenzaba a ser vista como un símbolo de la naciente contracultura, estableciendo de esta forma un posible guiño de identificación generacional entre The Beatles y sus fans (Fontenot, 2011).

\subsection{Acabado formal y soluciones técnicas innovadoras}

Finalmente, The Beatles trabajaban con sumo cuidado las melodías, explorando arreglos y soluciones alternativas que les permitiesen encontrar la mejor opción (The Beatles, 2000, p. 175) y cuidaban extraordinariamente la ejecución final, buscando un nivel de acabado perfecto, tal y como suelen hacer los compositores, arreglistas y productores de jingles publicitarios.

Con estos y otros recursos retóricos y musicales, The Beatles lograban la implicación y la complicidad del espectador, despertando su interés y tratando de crear sorpresa, igual que procuran hacer los jingles. Quizás esto explique, en cierta medida, el enorme éxito comercial del grupo y la larga vigencia de sus canciones, generación tras generación. Por su parte, algún autor ha señalado la similitud entre los jingles y la música pop, destacando el carácter ameno y el estilo fácil, sencillo y cercano de ambos géneros musicales (Fernández Gómez, 2008).

Además de las cuestiones puramente musicales y retóricas, The Beatles introdujeron soluciones técnicas que luego aplicaría la publicidad, como la compresión de las mezclas. Así, en los LPs "Revolver" y "Sgt. Peppers" el grupo decidió comprimir notablemente la mezcla final del sonido para darle brillo y claridad a las canciones

ICONO14 | Año 2013 Volumen 11 Nº 1 | ISSN: 1697-8293 | DOI: ri14.v11i1.558 
limitando los graves (The Beatles, 2000, p. 206). Este proceso sería utilizado posteriormente en las campañas de radio y televisión para subir el volumen de las piezas publicitarias sin distorsionar el contenido y es el responsable de que, en nuestro país, los bloques de anuncios tengan un volumen de emisión notablemente más elevado que el resto de la programación.

\section{Publicitando sus propias canciones}

Que se sepa, The Beatles nunca crearon expresamente música para acciones publicitarias. La única iniciativa que puede equipararse con ello fue una versión que realizaron para la emisora pública británica BBC de "From me to you", el tema que constituyó su tercer single como grupo y su primer número uno en la lista oficial de ventas británica (Record Retailer). Para ello, seleccionaron la estrofa inicial de la canción y cambiaron el texto del penúltimo verso ("with love from me to you", ver cuadro $\mathrm{n}^{0} 3-\mathrm{A}$ ) que fue sustituido por "with love from us to you") incorporando así el nombre del programa (From us to you) en el que fue radiada esta versión. La canción inicial tiene una duración de $1^{\prime}: 56^{\prime \prime}$, mientras que la nueva versión se recortó a $0^{\prime}: 27{ }^{\prime \prime}$.

\section{Cuadro 3a. Estribillo original de la canción "From me to you"}

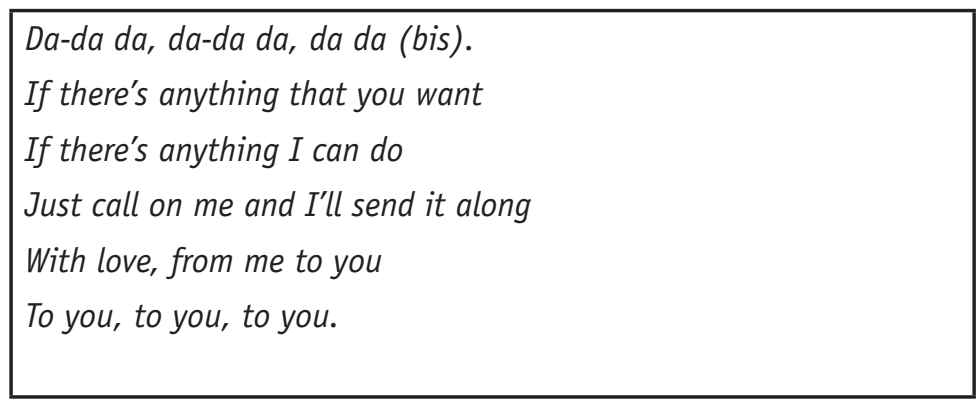

Fuente: The Beatles, 1994

Esta versión parece haber sido resultado de la improvisación, lo que constituiría otra demostración de la versatilidad, ingenio y capacidad de creación del grupo. Podemos decir que, como pieza de comunicación, constituye simultáneamente un 
anuncio de autopromoción del programa de radio, del tema musical utilizado y de los propios Beatles, cuando estos necesitaban presentarse porque el fenómeno social de la "beatlemanía" aún se estaba conformando. La pieza fue grabada el 28 de febrero de 1964 y se emitió en el citado programa From us to you en repetidas ocasiones, entre marzo y el verano de ese mismo año (Wikipedia, 2011).

Desde un punto de vista formal, este tema opera a la perfección como un jingle y cumple, a nuestro entender, todos los requisitos que definen a este tipo de piezas publicitarias, confirmando las relaciones de forma y fondo que hemos establecido anteriormente entre los jingles y un buen número de los temas más significativos de The Beatles.

Esta versión de "From me to you" puede escucharse en el disco recopilatorio "Live in the BBC" (ver The Beatles, 1994); en dicho CD esta canción ocupa el segundo lugar, precedida por una "autopresentación" denominada oficialmente "Beatles Greetings" que The Beatles habían grabado anteriormente también en la BBC, el 9 de octubre de 1963, y que fue emitida en el programa "The Public Ear" el 3 de noviembre de ese año (Wikipedia, 2011). Resulta muy llamativa la significación que adquieren estas dos grabaciones cuando se escuchan seguidas, tal como figuran en el CD, y no resulta en absoluto casual que hayan sigo elegidas y ubicadas en semejante orden para abrir esta amplia selección de canciones de los inicios del grupo.

En primer lugar, en la grabación "Beatles Greetings", los cuatros Beatles se presentan a sí mismos (ver cuadro $n^{0} 3-B$ ) dando su nombre y explicando el instrumento que tocan. Y lo hacen apelando al humor y a la naturalidad -Paul McCartney duda cuando habla, detrás de las voces se escucha un silbido, John Lennon hace un irónico juego de palabras- mostrando el mismo estilo desenfadado que luego exhibirían en sus conocidas ruedas de prensa (Miles, p. 47-69). Resulta paradójico pensar que, poco tiempo después y atendiendo al nivel de popularidad que el grupo alcanzaría a nivel mundial, una presentación de este tipo resultaría totalmente superflua, motivo que les llevaría a editar el primer disco publicado en la historia -"Rubber Soul"- que no mostraba en la portada el nombre del $\operatorname{artista}^{1}$ (Freeman, p. 62-64).

Tras esta presentación, comienza la nueva versión antes analizada de "From me to you". Y ambas piezas sonoras unidas - "Beatles Greetings" seguida de "From us to you"- recrean lo que podría habría sido una perfecta cuña publicitaria del grupo 


\title{
Cuadro 3b. Texto de los dos primeros temas de "Live in the BBC"
}

\author{
Ringo: I'm Ringo and I play the drums. \\ Paul: Hey... I'm Paul and I play the... hum... bass. \\ George: I'm George and I play a guitar (whistle). \\ John: I'm John and I too play guitar... Sometimes I play the fool (1). \\ The Beatles: Da-da da, da-da da, da da (bis) \\ If there's anything that you want \\ If there's anything we can do \\ Just call on us and we'll send it along \\ With love, from us to you \\ To you, to you... \\ Ringo: Soy Ringo y toco la batería \\ Paul: Hola... Soy Paul y toco el... este... el bajo. \\ George: Soy George y toco una guitarra (silbido). \\ John: Soy John y también toco la guitarra... Y a veces hago el loco ${ }^{(1)}$. \\ The Beatles: La-la la, la-la la, la la (bis) \\ Si hay algo que deseas \\ Si hay algo que podamos darte \\ Basta con que nos llames y te lo enviaremos \\ Con mucho amor, de nosotros para tí
}

(1) Un juego de palabras aprovecha el doble sentido del término "play", que aquí es utilizado en sus acepciones de "tocar un instrumento" e "interpretar un papel".

Fuente: The Beatles, 1994

de 41 segundos de duración y desvela los valores que ambas grabaciones por separado tienen como piezas de promoción comercial y de comunicación persuasiva, muy adelantadas a su tiempo.

Debemos mencionar que el recurso de utilizar el estribillo de una canción cambiando parcialmente la letra fue copiado posteriormente un gran número de veces. En nuestro país, durante los años 80 , se utilizaba muy a menudo para que dife- 
rentes grupos y cantantes de éxito autopromocionasen sus canciones en "Los 40 Principales" que, por entonces, era un programa de la Cadena Ser y no una emisora de radio.

\section{Presencia de la música beatle en grandes}

\section{campañas}

Aunque sus ideas y su música fueron muy imitadas en la producción sonora de campañas publicitarias, The Beatles nunca permitieron licenciar el copyright de sus canciones ni su propia imagen para utilizarlas en campañas de publicidad, siguiendo el mismo criterio que otras grandes figuras de su época. Bob Dylan, por ejemplo, ha manifestados repetidas veces su negativa a acciones de este tipo, a pesar de tener ofertas millonarias, aunque posteriormente intervino en un anuncio de los automóviles Cadillac (Wheaton) y promocionó la Exposición Mundial de Zaragoza celebrada en 2008 (Dylan, 2007 y 2008).

Esta situación sufrió un cambio drástico cuando el conocido cantante y compositor Michael Jackson compró en 1985 los derechos de las canciones de The Beatles (ver Crawford) y para rentabilizar esta inversión comenzó a licenciar sus derechos autorales con fines publicitarios. España fue uno de los primeros países en sacar partido de ello, utilizando canciones de The Beatles en una campaña de la marca de refrescos Kas -perteneciente al grupo PepsiCo Inc.- realizada por la agencia de publicidad Danis, Benton \& Bowles (empresa hoy absorbida por la red de Leo Burnett Woldwide). Poco después, un cover de "Imagine" prestaba su música a un anuncio de Rank Xerox ("Imagine un mundo sin problemas") realizado por la agencia española Contrapunto (hoy Contrapunto/BBD0) y premiado en varios festivales internacionales de publicidad.

Igualmente, una campaña norteamericana de Nike utilizó la canción original de "Revolution" en 1987 aprovechando un vacío legal (ver Nike 1987) contra los deseos expresos de Yoko 0no, persona depositaria de los derechos de la obra artística de John Lennon. Posteriormente esa misma marca utilizaría la versión original de "Instant Karma" en una campaña realizada por la agencia Wieden+Kennedy (ver Nike 1993) pero, esta vez, con la autorización de la viuda de Lennon. Igualmen- 


\title{
Cuadro 4. Texto del spot "God" realizado para Golf
}

\author{
John Lennon: I don't believe in Elvis. (Subtítulo: No creo en Elvis) \\ I don't believe in Kennedy. (Subtítulo: No creo en Kennedy) \\ I don't believe in Beatles. (Subtítulo: No creo en Beatles) \\ I just believe in me. (Subtítulo: Sólo creo en mí) \\ Locutor: ¿Y tú? ¿Crees en Golf?
}

Fuente: Golf,1998

te, Yoko Ono negoció personalmente el uso del tema "God" con la productora de jingles publicitarios "Sintonía" (Espadalé, p. 289) para que fuese utilizado en una campaña del automóvil Golf realizada en nuestro país por la agencia DDB Barcelona en 1998 (ver Golf, 1998). El mensaje de este anuncio puede leerse en el cuadro $n^{0}$ 4.

Estos hechos nos permiten comprobar que, con el paso del tiempo, la música de The Beatles no se limitó a influir en las características de la producción musical publicitaria sino que incluyo llegó a suplantarla, precisamente en el momento en el que los jingles comenzaban a ser sustituidos por canciones originales, versiones o covers de las mismas, como hemos mencionado antes (Espadalé, p. 289). Esto ocurre en una reciente campaña de Movistar, que utiliza como jingle una versión de "Come together" de Lennon, que los usuarios de esa operadora telefónica interpretan con los sonidos de sus teléfonos móviles (ver Movistar 2012).

\section{Conclusión}

En este trabajo hemos podido demostrar, según creemos, la influencia y la clara vinculación que la producción musical de The Beatles ha ejercido sobre los jingles publicitarios, gracias al análisis de las figuras y recursos retóricos presentes en la misma. Igualmente, hemos analizado la única composición realizada por este grupo con un fin intencionadamente publicitario. Y también hemos detallado algunos transvases existentes entre la música popular y la actividad publicitaria, analizando someramente los casos concretos que nos han ofrecido artistas y campañas. 
Ello nos ha permitido comprender mejor estas conexiones, pero quizás nos permita además abrir otra vía de debate en un tema de notable trascendencia social que, aunque ya ha sido estudiado, está lejos de agotarse y que comprende importantes consideraciones semióticas. Nos referimos a la transferencia de valores existente entre la música popular, sus ídolos y las campañas publicitarias y otras acciones de comunicación persuasiva.

\section{Discusión. Del uso de la música a la apropiación semántica de la imagen}

Excediendo el mero terreno de la utilización musical, en épocas más recientes hemos asistido al uso y explotación de la imaginería misma de The Beatles por parte de campañas publicitarias. La campaña "Think Different" (ver Apple, 1997), realizada para Apple por la agencia TBWA/Chiat/Day y reconocida con importantes premios a nivel mundial por su planteamiento estratégico y creativo, mostraba a John Lennon acompañado de Yoko Ono durante su famoso "Bed-In". En el anuncio de televisión, Lennon aparecía en un fragmento junto a otros personajes famosos configurados como modelos sociales de amplia aceptación, cuya obra y actitud había logrado hacer evolucionar el establishment de forma positiva. La imagen

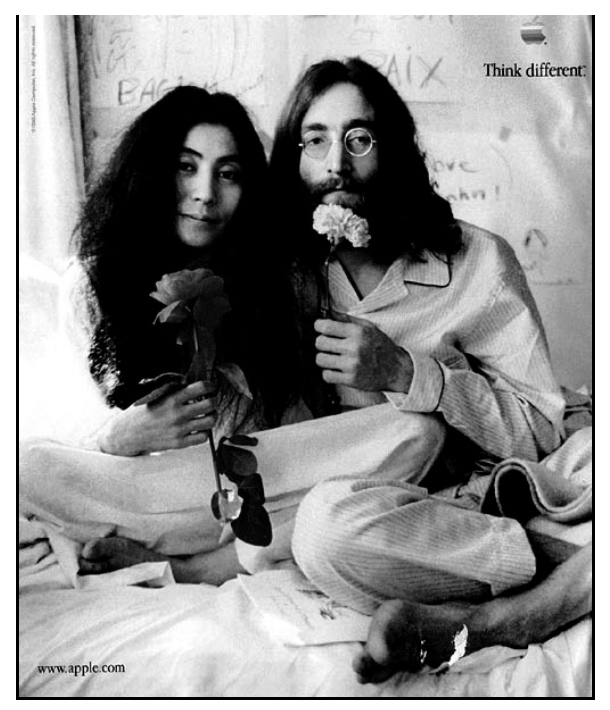

\section{Gráfico 2}

La famosa "Bed-In" o protesta en la cama, realizada por Lennon y Ono como un gesto por la paz, utilizada en los carteles de la campaña de Apple "Think Different". 
fotográfica de Lennon y Ono merecía un papel protagonista en carteles y piezas de publicidad exterior utilizadas en todo el mundo, incluyendo nuestro país (ver gráfico $n^{0} 2$ ). En el repertorio de piezas de esta campaña aparecían otros cantantes de diferentes géneros musicales, populares y cultos, como Bob Dylan, Bono (U2), Frank Sinatra o María Callas.

Esta utilización estelar del miembro más inconformista de The Beatles realizada por Apple, se alinea perfectamente con la estrategia de marketing y comunicación de esta marca, posicionada desde hace tiempo como una empresa heterodoxa y revolucionaria frente al -por oposición-convencionalismo de los $P C$ y de las demás marcas operantes en el sector de la informática y la tecnología.

Ello subraya con nítida claridad un hecho que con el tiempo se ido configurando de manera evidente: la comunicación publicitaria ha comenzado a utilizar la música de artistas y grupos famosos no sólo por sus adecuación para ilustrar musicalmente ciertas campañas o por sus valores intrínsecos para promocionar una determinada marca. Lo ha hecho también y sobre todo, como un mecanismo para apropiarse de aspectos de la personalidad de esos artistas, de los valores que representan y, por extensión, del espíritu de los públicos a los que se dirigen y para los que constituyen un referente aceptado. Admitiremos entonces que este proceso implica un mecanismo de deslizamiento semántico pues, partiendo de la música, la comunicación publicitaria ha llegado a servirse de la imagen y de los valores proyectados por ciertos artistas, con el objetivo de conectar con y merecer la credibilidad de los públicos seguidores de dichos artistas ya que, como sabemos, el contenido expresado en un anuncio revierte sus propiedades hacia el producto anunciado (Saborit, p. 89) y posteriormente hacia la marca que lo ampara. Lógicamente, se trata de un proceso generalmente consentido e incluso propiciado por artistas y grupos de música, que han encontrado en esta asociación comercial -cuando se realiza correctamente, alineando los intereses de las marcas y de los artistas- una vía para incrementar su popularidad y para contar con un canal adicional que propague y glamourice su música ante audiencias masivas.

Sin duda, este es un tema interesante, amplio y lleno de matices, aunque complicado de abordar tanto por su complejidad y como por su transversalidad. Es un debate que queda abierto y en el que hemos querido aportar algunos elementos a los que hemos llegado, como hemos visto, partiendo de la música popular y de su utilización por parte de la publicidad. Pero podemos concluir añadiendo que esta

DOI: ri14.v11i1.558 | ISSN: 1697-8293 | Año 2013 Volumen 11 No 1 | ICONO14 
apropiación semántica de lo popular y de los valores sociales y estéticos encarnados por los ídolos de moda, no es privativa de la actividad publicitaria: también podemos presenciarla en las campañas políticas ${ }^{2}$ (Berlinger), en la práctica profesional de las relaciones públicas y en otras muchas actividades de la comunicación persuasiva.

\section{Agradecimientos}

Deseamos agradecer a varios profesionales de la publicidad y de la producción musical su ayuda documental para este artículo, especialmente a José Carlos Mac de Thebe Mor Creative Studio, Lettoch, Escocia, Reino Unido.

\section{Notas}

[1] Robert Freeman, fotógrafo oficial de las portadas de los Beatles de 1963 a 1965, ha confirmado al autor de este artículo que tanto The Beatles como él intentaron que el nombre del grupo no apareciese en la portada del disco "Help". No pudieron convencer de ello a la casa discográfica EMI, aunque consiguieron hacerlo en el siguiente disco, "Rubber Soul", publicado solo seis meses después.

[2] Véase hacia el final de esta obra documental, las declaraciones del músico Paul Simon sobre la importancia de la utilización de celebrities de la música popular por parte de los políticos. Paul Simon apoyó a George McGovern en la campaña de las elecciones presidenciales de EEUU (1972) y se posicionó contra el apartheid en Sudáfrica durante la gira de su CD "Graceland" (1986-7).

\section{Referencias}

Álvarez, A. (2003). La función social de la publicidad de la Once. Madrid: Escuela Libre Editorial-Fundación ONCE.

Aizcorbe, X. (2011). Otros imaginarios, otras músicas. La música en la publicidad: un arma para llegar al alma. Barcelona: Universitat Abat Oliba-CEU. Recuperado el 31/enero/2013, 17:30h. en: http://www.recercat.cat// handle/2072/178859

ICONO14 | Año 2013 Volumen 11 № 1 | ISSN: 1697-8293 | DOI: ri14.v1 1i1.558 
Apple (1997). Campaña “Think Different” . Recuperado el 31/enero/2013,

17:30h. en: http://www.youtube.com/watch?v=40AB83Z1ydE

Berlinger, J. (Director). (2012). Under African Skies. Paul Simon's Graceland Journey. New York, USA: A\&E Indie Films.

Crawford, K. (2005). Michael Jackson to lose Beatles catalog? en CNN Money.com, 8 de junio. Recuperado el 31/enero/2013, 17:35 h. en: http://goo.gl/bUiam Cola-Cao (1956). Anuncio de televisión que utiliza una versión el famoso jingle.

Recuperado el 17/enero/2013, 7:35h. en: http://goo.gl/2RQIv

Dister, A. (1983). Los Beatles. Madrid: Ediciones Júcar.

Dylan, Bob (2007). Campaña para Cadillac Escalade. Recuperado el 31/

enero/2013, 17:35h. en: http://www.youtube.com/watch?v=9X3Bcmf3ckQ

Dylan, Bob (2008). Campaña de publicidad para promocionar la Exposición

Mundial de Zaragoza, spot de televisión. Recuperado el 31/enero/2013,

17:35h. en: http://goo.gl/FGXrw

Espadalé, B. (2000). El sonido de la publicidad, en Moliné, Marçal (editor), La fuerza de la publicidad. Madrid: McGraw Hill.

Fernández Gómez, J. D. (2002). Hacia una funcionalidad de la música en el spot publicitario. Revista Internacional de Comunicación Audiovisual, Publicidad y Estudios Culturales, ${ }^{0} 1$, páginas 161-178. Sevilla: Universidad de Sevilla.

Fernández Gómez, J. D. (2008). Música, publicidad y juventud: Sonic Youth, en Etayo, C. y otros (editores), Los jóvenes y el nuevo escenario de la comunicación: actas del XXI Congreso Internacional de Comunicación:

Ediciones Universitarias de Navarra-EUNSA, Pamplona. P. 1-10.

Ferrer, E. (1994). El lenguaje de la publicidad. México: Fondo de Cultura Económica.

Freeman, R. (1990). The Beatles, a private view. New York, USA: Mallard PressBDD Promotional Book Company.

Fontenot, R. (2011). The Beatles Songs: Girl. The history of the classic Beatles song. About.com Oldies Music. Recuperado el 31/enero/2013, 17:30h. en: http://goo.gl/sS74S

Golf (1998). Anuncio "God" con música del tema de John Lennon del mismo título. Recuperado el 31/enero/2013, 17:30h. en: http://goo.gl/L7v6u

Hoyuela, P. y Lázaro, Y. (2003). Diccionario J, Walter Thompson. Comunicación,

DOI: ri14.v11i1.558 | ISSN: 1697-8293 | Año 2013 Volumen 11 Nº 1 | ICONO14 
Marketing y Nuevas Tecnologías. Madrid: Ediciones de las Ciencias Sociales, S.A.

Lorente, J. (1995). Casi todo lo que sé de publicidad. Barcelona: Editorial Folio. Marlboro (1963). Anuncio con el tema musical de "Los Siete Magníficos".

Recuperado el 16/junio/2011, 16:00h. en: http://www.youtube.com/ watch?v=1UQY7srgec8

Marlboro (1967). Anuncio de esta marca promocionando un disco con la música de sus campañas. Recuperado el 31/enero/2011, 17:40h. en: http://goo.gl/ zP8sf

McCartney, Paul (2003). Interpretación del tema "She's leaving home" el 24/ mayo/2003 en la Plaza Roja de Moscú. Recuperado el 31/enero/2013, 17:40h. en: http://goo.gl/fUkEW

Miles, B. (compilador). (1978): Los Beatles vistos por sí mismos. Madrid: Ediciones Júcar.

Movistar (2012). Campaña "Movistar Fusión". Recuperado el 31/enero/2013, 17:30h. en: http://www.youtube.com/watch?V=VDVC-04R9NI

Muela, C. (2007). La estrategia creativa en la cuña radiofónica con fines sociales. Análisis del mensaje en el medio radio: el contenido y la forma. Revista Creatividad y Sociedad, $\mathrm{n}^{0}$ 11, octubre. Madrid: ASOCREA. Recuperado el 31/ enero/2013 17:40h. en: http://goo.gl/LnPi7

Nike (1987). Campaña con el tema “Revolution" (16/junio/2011, 14:45h.): http://www.youtube.com/watch?v=iMXhtFik-vI

Nike (1993): campaña con el tema “Instant Karma". Recuperado el 31/ enero/2013, 17:45h. en: http://goo.gl/ny0sF

Once-Organización Nacional de Ciegos (1983). Primera versión del jingle de la Once. Recuperado el 31/enero/2013, 17:45 h. en: http://goo.gl/FEHU6 Palencia-Lefler, M. (2009). La música en la comunicación publicitaria. Revista Comunicación y Sociedad, volumen XXII, $n^{\circ} 2$, p. 89-108. Recuperado el 31/ enero/2013, 17:45h. en: http://goo.gl/1yL5a

Sala, J. (2009). Del jingle a las canciones pop: una aproximación al uso de la música en la publicidad. Barcelona: Universitat Abat Oliba-CEU. Recuperado el 13/enero/2013, 16:50 h. en:http://www.recercat.net/handle/2072/39533

Sánchez Guzmán, J. R. (1993). Teoría de la Publicidad. Madrid: Editorial Tecnos. Saborit, J. (2012). La imagen publicitaria en televisión. Madrid: Ediciones 


\section{Cátedra.}

Simon, P. (1966). "The Big Bright Green Pleasure Machine", tema del LP Parsley, Sage, Rosemary and Thyme. EEUU: Columbia Records-CBS.

Souter, N. y Newman, S. (1988). Creative Director's Sourcebook. London:

MacDonald \& Co.

Sturges, J. (1960). The Magnificent Seven. USA: United Artists.

Summers, G. (2000). Yo soy aquel negrito. Los anuncios que marcaron nuestra vida. Barcelona: Ediciones Martínez Roca.

The Beatles (1965) Actuación interpretando la canción "Help!" Recuperado el 31/ enero/2013, 18:30h. en: http://goo.gl/KHRXB

The Beatles (1994). Canción "From us to you" del CD Live at the BBC. USA:

Capitol.

The Beatles (2000). The Beatles Antología. España: Ediciones B.

The Beatles Bible (2011). Recuperado el 31/enero/2013, 17:45h. en: http://goo. $\mathrm{gl} / \mathrm{sAaOI}$

U2 (1993). "Zooropa”, tema del CD del mismo nombre. United Kingdom: Island Records.

Wheaton, K. (2007). “Bob Dylan Shills for Cadillac". Advertising Age, 22 de octubre. Recuperado el 31/enero/2013, 17:50h. en: http://goo.gl/7o00C Wikipedia (2010). Recuperado el 31/enero/2013, 17:50h. en: http:// en.wikipedia.org/wiki/Jingle Wikipedia (2011). Recuperado el 31/enero/2013, 17:50h. en: http://goo.gl/LIy9 